\title{
掺 $\mathrm{Cu}^{+}$玻璃荧光的格位效应
}

\author{
刘慧民千福孰 \\ (中国科学院上海光学精密机拢研究所)
}

无机玻璃中由于顺磁离子所处格位经受着对角和非对角渏变, 导致了它的光谱和 ESR 谱 线都有严重的不均匀加宽.它导致格位与格位之间能级本征值的畸变以及耦合强度的差别. 在 研究玻璃中掺杂离子的发光性质时, 它的发光机制时常被这种扩展对称性所引起的效应所掩 没. 因此利用选择激发和零延迟取样技术来揭示不同格位苂光光谱分布是十分有意义的。

\section{一、吸收 光谱}

图 1 是氟磷酸盐玻璃中 $\mathrm{Cu}^{+}$的吸收光谱。玻璃的化学成分 为 (mole\%): $\mathrm{P}, \mathrm{O}, 5.55$, $\mathrm{NaPO}_{3} 7.49, \mathrm{MgF}_{2} 16.45, \mathrm{CaF}_{2} 16.34, \mathrm{SrF}_{2} 12.16 \mathrm{BaF}_{2} 11.63, \mathrm{AlF}_{3} 30.39$, 外加 $\mathrm{NaPO}_{3} 3.0$.

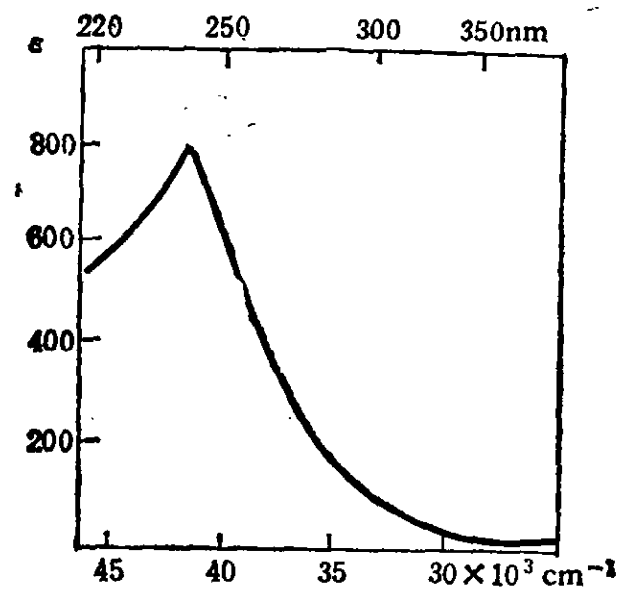

因 1 室温下舁磷酸盐玻玹中 $\mathrm{Cu}^{+}$的吸收光谱

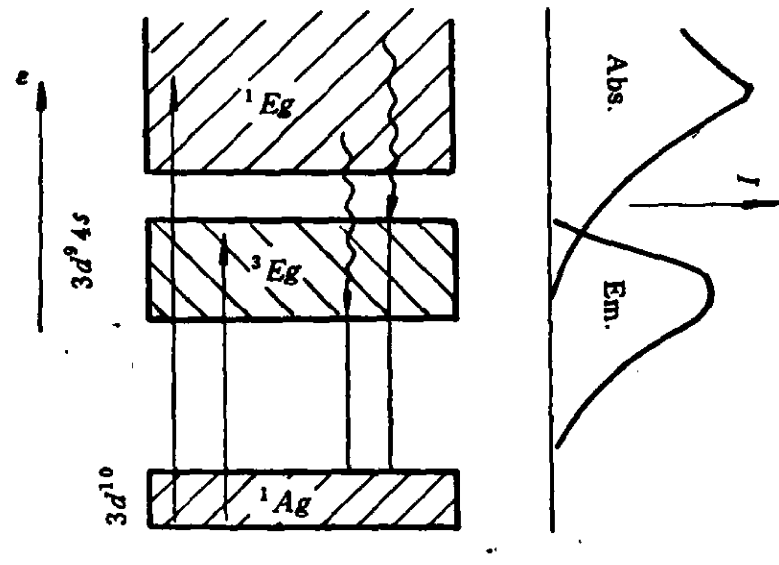

图 2 扩展 $\mathrm{O}_{\mathrm{b}}$ 对称性格位中的 $\mathrm{Cu}^{+}$的能级本 征值綺变和声子支助发射模式 它是引起吸收和发射谱中宽带结构的根獂

吸收谱峰值位于 $41300 \mathrm{~cm}^{-1}$. 文献 [1] 已说明它是属于 Laport 禁戒的 $d^{10} \rightarrow d^{9} s$ 组态间跃 迁. 但光谱谱线有相对较强的振子强度. 这可能起因于格位偏离于完美立方对称性. 从而导 致选律的松驰. 如以下将要讨论的驰豫谱中可以确信, 吸收光谱可指定为属于 $\mathrm{O}_{\mathrm{h}}$ 对称性格位 中 $\mathrm{Cu}^{+}$的 ${ }^{1} \mathrm{~A}_{\mathrm{1g}}\left(3 d^{10}\right) \rightarrow{ }^{1} \mathrm{E}_{\mathrm{g}}\left(3 d^{9} 4 s^{1}\right)$ 跃迁。其谱线的宽带结构除了有部分 ${ }^{1} \mathrm{~A}_{\mathrm{ig}} \rightarrow{ }^{3} \mathrm{E}_{\mathrm{g}}$ 跃迁的贡献 （见图 2), 以及吸收主带与 $d \rightarrow p$ 跃迁的带边发生重迭, 从而导致线宽增加外, 主要是来源于 格位的不均匀分布.

对于 $\mathrm{Cu}^{+}, \mathrm{Na}^{+}$两种离子，它们既有相同的价态，又有相近的离子半径. $\mathrm{Cu}^{+}$为 $0.96 \AA$ ， $\mathrm{Na}^{+}$为 $0.95 \AA$. 因此, 在玻璃网络中, $\mathrm{Cu}^{+}$离子可取代 $\mathrm{Na}^{+}$而成为填隙离子. 它可与桥等钱 合, 也可供作电荷补偿. 这时, $\mathrm{Cu}^{+}$离子格位的对称性是高度畸变的.

本文 1987 年 2 月 23 日收到. 


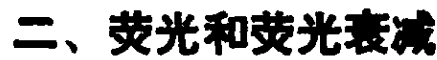

图 3 代表少磷酸盐玻琌中 $\mathrm{Cu}^{+}$在室温和 $77 \mathrm{~K}$ 下的荻光光谱。实验中的光源采用高压窄 脉冲缶灯。激发波长为 $250 \mathrm{~nm}$ ，带宽 $10 \mathrm{~nm}$ 。荧光信号用 Boxcar Averager 采集。激发后,立 即在苂光信号的前沿上打开取样门. 门宽设定为 $2 \sim 5 \mu \mathrm{s}$. 用这种方法(所谓无延迟取样)可 以有效地消除由于能量转移等时间有关过程所引起的谱形变化，并获得原始受激格位的发光 光谱(但无辐射驰豫因其过程十分迅速,影响依然存在).

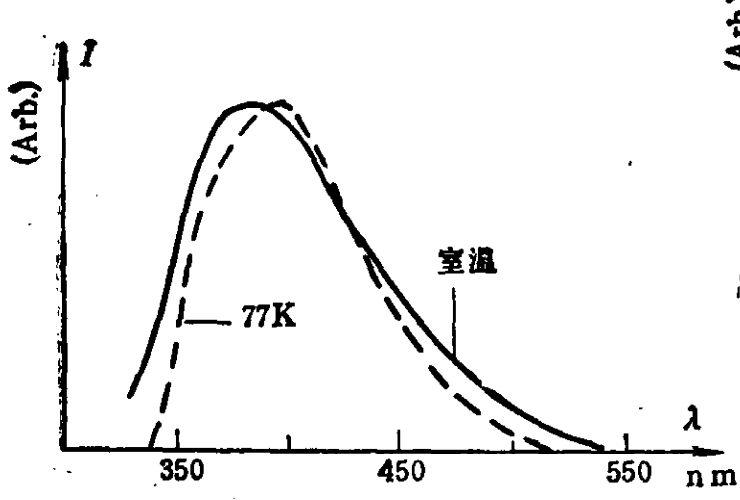

因 3 不同温度下策磷酸盐玻琌中 $\mathrm{Cu}^{+}$的荧光光谱

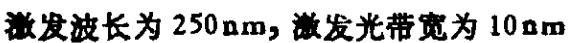

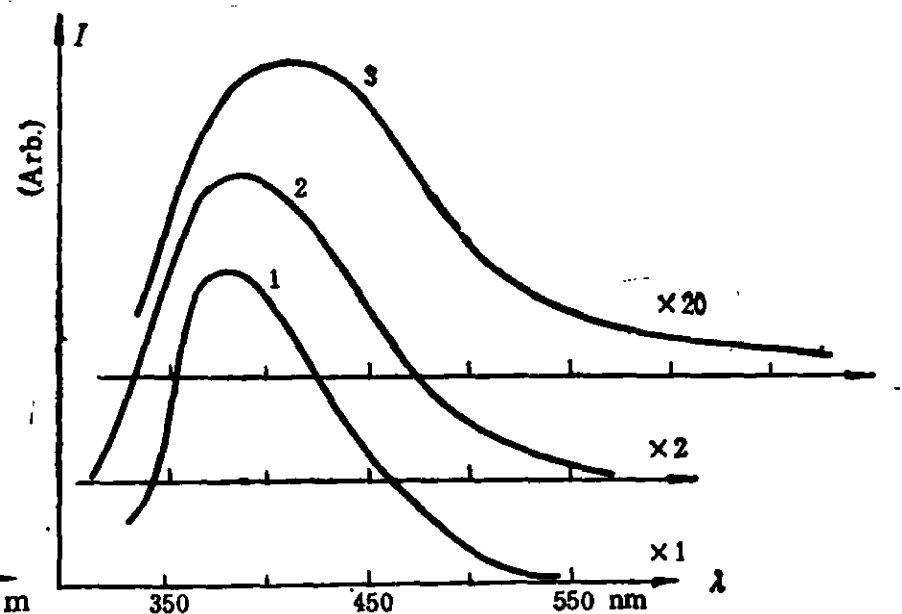

图 4 室温时,不同波长激发下氮磷酸盐玻璃中 $\mathrm{Cu}^{+}$的荧 光光谱各㙕线强度归一化，右边数字为增益

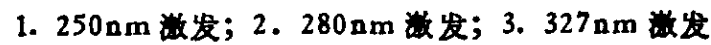

如图所示, 不同温度下的光谱线形和带宽十分相似。室温下峰值位置在 $380 \mathrm{~nm}, 77 \mathrm{~K}$ 时 在 390nm. 对于这种因温度升高所引起发射波长的兰移已在文献 [2] 中作过讨论. 这种现象 在过渡金属离子掺杂的玻璃中是常见的 ${ }^{[2,3]}$. 除此以外, 图 3 中两条谱线无大差别. 这说明不 同温度下激发在同一波长时,只有具有相近格位对称性的那些 $\mathrm{Cu}^{+}$离子才会被激发,并表现出 相近的光谱外形. 相反,图 4 是同一温度下,不同波长激发时 $\mathrm{Cu}^{+}$的苂光光谱. 谱线也由无延 迟取样法记录. 因此它们也各代表着原始受敫离子格位的光谱分布. 图中曲线 $1-3$ 分别表 示激发波长为 250,280 和 $327 \mathrm{~nm}$ ，教发带宽为 $10 \mathrm{~nm}$ 的苂光光谱. 它们的峰值位量 2 和线 宽(FWHM) $\Delta \lambda$ 为

$$
\begin{aligned}
& \lambda_{250}=380 \mathrm{~nm}, \quad \Delta \lambda=90 \mathrm{~nm} ; \\
& \lambda_{280}=385 \mathrm{~nm}, \quad \Delta \lambda=105 \mathrm{~nm} ; \\
& \lambda_{3 z}=415 \mathrm{~nm}, \quad \Delta \lambda=132 \mathrm{~nm} .
\end{aligned}
$$

这说明随着激发波长移向长波,受激离子的辐射能量降低, 其格位的光谱分布区域逐渐加宽. 我们曾试图采用比 $250 \mathrm{~nm}$, 即比㳓光谱的蜂值位置 ${ }^{[4]}$ 更短波长激发来观察茨光光谱. 然而 如所预料的,其结果与采用 $250 \mathrm{~nm}$ 散发所得到的光谱相同. 这是因为玻璃基质中过渡元素离 子的辐射衰减是一定,也必须有声子的发射相伴随 ${ }^{[5]}$, 即所谓声子支助的辐射跃迁. 这种跃迁 方式已表示在图 2 中.

表 1 是不同温度下氮磷玻琌中 $\mathrm{Cu}^{+}$的苂光寿命与激发波长和发射波长的关系。值得注意 的是整个荧光衰减期间达到数十以至数百微秒。因此，与无延迟激发方式不同的是这里所得 
到的荧光衰减曲线包含了格位间能量转移的贡献. 特别是那些具有较长发射波长的离子, 它 们在能量转移过程中扮演了受体的角色. 因此实验测定的荧光寿命被增加很多.

表 1 不同温度下,氧磷酸盐玻璃中 $\mathrm{Cu}^{+}$在不同激发和发射波长时的荧光寿命数据 ( $\left.\mu \mathrm{s}\right)$

\begin{tabular}{|c|c|c|c|c|c|c|c|c|c|c|c|}
\hline \multirow{2}{*}{\multicolumn{2}{|c|}{$\frac{\text { 温 度 }}{\text { 擞发波长 }(\mathrm{nm})}$}} & \multicolumn{3}{|c|}{ 室 } & \multicolumn{2}{|l|}{ 温 } & \multicolumn{5}{|c|}{$77 \mathrm{~K}$} \\
\hline & & 360 & 420 & 500 & 560 & 640 & 360 & 420 & 500 & 560 & 640 \\
\hline \multirow{4}{*}{$\begin{array}{c}\text { 橴发波长 } \\
(\mathrm{nm})\end{array}$} & 220 & (27) & (36) & $(50)$ & $(60)$ & $(80)$ & (29) & (43) & $(60)$ & & \\
\hline & 250 & 33 & 37 & 42 & 48 & & (37) & 52 & 72 & 88 & 115 \\
\hline & 280 & 35 & 43 & 62 & 84 & 90 & 40 & 55 & 85 & 125 & 130 \\
\hline & 330 & & 44 & 62 & 106 & 114 & 41 & 55 & 95 & 135 & 145 \\
\hline
\end{tabular}

如表 1 所示, 对一定的发射波长(亦即检测波长固定), 茨光衰减随激发波长而变. 也就是 说, 一定的激发波长就相对应于不均匀加宽体系中的某一组格位离子. 当激发波长移向长波 时, 荧光寿命就增加. 另一方面, 对一定的激发波长, 荧光衰减速度强烈地依赖于体系所设定 的检测波长位置. 与以上结果相平行, 发射波长愈长, 所测得的寿命也愈长, 它们彼此间可相 差数倍.

此外, 比较一下室温下和 $77 \mathrm{~K}$ 下表 1 中的光谱数据可以看到它们都有一相似的变化趋 势. 说明只要最初受激格位的分布不变,温度对光谱的影响并不显著.

根据著名的 Fuchtbane-Ladenburg 方程 ${ }^{[6]}$, 可以预期对自旋禁戒的 ${ }^{3} \mathrm{E}_{\mathrm{g}} \rightarrow^{1} \mathrm{~A}_{\mathrm{ig}}$ 跃迁，将有 长得多的荻光寿命. 然而实际所测得的寿命要短十倍以上. 其原因可能如同吸收谱有较高的 振子强度一样,它起因于组态相互作用导致从自旋允许跃迁中发生几率偷窃.

最后,表 1 中所列 $220 \mathrm{~nm}$ 激发数据加一括号是表示发光衰减呈非指数式. 这是由于能岳 转移发生交叉驰豫而引起, 将在以后另作讨论.

\section{三、结 论}

在掺 $\mathrm{Cu}^{+}$氧磷酸盐玻璃中,杂质离子在基质网络中起着填隙离子的作用. 其所处格位经 受着不同程度的畸变. 也就是说，存在着格位对称性的扩展. 利用较窄的脉冲数发和不同数 发波长,可以使得仅仅一小组格位,它们偏离正规八面体对称性的程度非常相近，从而被选择 激发到 $d^{9} s$ (或 $d^{9} p$ ) 态. 采用无延迟取样技术消除能量转移的影响,使得这一定格位的光谱性 质被清楚地呈现出来. 具有较低辐射能量的离子有较宽的光谱分布区域，但权重是偏于整个 谱带的短波处. 发射波长愈长,荧光衰减速率愈小,则寿命就愈长.

[1] Park, S. \& Webb, R. S., Phys. Chem. Glasses, 13(1972), 157.

[2] 刘慧民、干福意, 科学通报, 30(1985),9: 666 .

[ 3 ] Park, S., J. Phys. Chem. Solids, 32(1971), 669.

[4] 刘䉥民福言, 发光与显示, 5(1984), 1 .

[5] Liu Huimin and Gan Fuxi, J. Non-Crysz. Solids, 80(1986), 422.

[6] Park, S. and Watson, A. I., Phys. Chem. Glasses, 10(1969), 37. 